\title{
Das Parlament zum Thema Qualität
}

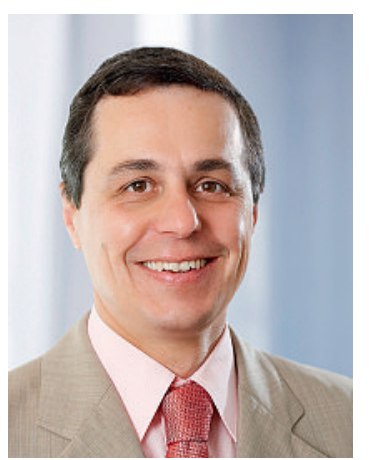

Die KVG-Reform 09.053 «Massnahmen zur Eindämmung der Kostenentwicklung», die der Bundesrat im Mai 2009 als Reaktion auf die Prämiensteigerungen eingereicht hat, ist gescheitert. Mit 97 Nein- zu 76 Ja-Stimmen hat eine Allianz von SP und SVP - bei Stimmenthaltung der Grünen - diese Minireform im Nationalrat (NR) bachab geschickt. Die Logik hinter dieser Kehrtwende hat nur wenig mit dem Inhalt der Reform zu tun: Der Walliser Sozialdemokrat Stéphane Rossini gab bekannt, seine Fraktion werde die Reform nicht unterstützen. Die SVP interpretierte das als Taktik, um den Druck auf das Gesundheitssystem aufrechtzuerhalten und um sich als Vertreter der Patienteninteressen zu profilieren. Deshalb drückte die SVPFraktion auf den roten Knopf und machte damit - in 20 Sekunden - die Arbeit von eineinhalb Jahren zunichte. Es lebe die Schweiz!

Die Reform enthielt auch interessante Vorschläge wie etwa den neuen Artikel zur Qualität [1]. Die Qualität ist nach wie vor ein wichtiges Thema, sowohl auf beruflicher als auch auf politischer Ebene. Es ist ein wenig wie mit der Bibel: Alle zitieren sie, doch nur wenige haben sie gelesen. Die Definition der Qualität ist unklar, und Qualität lässt sich leicht für andere Zwecke instrumentalisieren.

\section{Mehrere parlamentarische Vorstösse verlangen die Schaffung eines nationalen Qualitätsinstituts}

Unabhängig vom abgelehnten Paket stellt sich die Frage, wie es um die Qualität im Gesundheitssystem steht. Im Zusammenhang mit dem Inkrafttreten des KVG wurde sie 1996 zum ersten Mal ausdrücklich im Gesetz erwähnt (Art. 58). Mit einer liberalen Haltung hatte es der Gesetzgeber den Tarifpartnern (Ärzteschaft und Versicherern) überlassen, die Qualitätsverfahren bilateral festzulegen: Damit wollte er für sie einen uneingeschränkten Spielraum gewährleisten. Zehn Jahre danach wollte das Parlament wissen, wie es um den Fortgang der Arbeiten steht, und beauftragte die Geschäftsprüfungskommission (GPK), dies zu beurteilen. Die GPK veröffentlichte am 13. November 2007 den Bericht «Evaluation über die Rolle des Bundes bei der Qualitätssicherung nach KVG» [2]. Fazit: Die Situation ist unbefriedigend! Das Qualitätsmanagement erfolgt nur fragmentiert, diskontinuierlich und ohne Sicht auf das Ganze. Darum beschloss das Parlament, dem Bundesrat die Führung im Qualitätsbereich zu übertra- gen. Am 18. Juni 2008 erklärte sich dieser dazu bereit [3]. Am 9. Oktober 2009 unterbreitete er die Qualitätsstrategie des Bundes im Schweizerischen Gesundheitswesen [4]. Seither arbeitet er - über das BAG - mit mehreren Akteuren, insbesondere der FMH, an der Umsetzung dieser Strategie. Gleichzeitig wurden verschiedene parlamentarische Vorstösse eingereicht: die parlamentarische Initiative Heim (07.486), die von der Kommission für soziale Sicherheit und Gesundheit des NR (SGK-N) in eine Motion umgewandelt wurde (10.3015); die Motion SGK-S (Sommaruga, 10.3353) und die Motion der freisinnig-demokratischen Fraktion (Cassis, 10.3450). Am 28. September 2010 hiess der Nationalrat die Motion 10.3015 mit grosser Mehrheit gut. Diese parlamentarischen Vorstösse verlangen die Schaffung eines nationalen Qualitätsinstituts, das steuern, koordinieren und vereinheitlichen soll. Derzeit laufen die Vorbereitungen für ein solches Institut.

\section{Das Kürzel HTA und der Begriff Medical Board gehören allmählich zum politischen Vokabular}

Auch die Wirtschaftlichkeit von medizinischen Leistungen wird debattiert. Das Kürzel HTA (Health Technology Assessment) und der Begriff Medical Board gehören allmählich zum politischen Vokabular. Die Motion der FDP-Fraktion (Cassis, 10.3451) verlangt eine nationale HTA-Agentur. Der Bundesrat hat diese Motion bereits befürwortet, das Parlament hat sich noch nicht mit ihr befasst. HTA wird neben der Qualität schon bald ein beliebtes Kürzel sein. Nun ist noch die Verbindung zwischen der Politik und den Fachleuten zu schaffen, um zu vermeiden, dass sich diese Themen auf zwei parallelen Schienen entwickeln, die sich ignorieren oder sogar bekämpfen! Die Abteilung DDQ setzt sich dafür ein, diese Verbindung zu gewährleisten.

Dr. med. Ignazio Cassis Vizepräsident der FMH und Nationalrat

Weitere Informationen zu den angegebenen Geschäftsnummern finden Sie auf www.parlament.ch.

1 Art. 43 6bis (neu). Versicherer können in Verträgen mit Leistungserbringern, welche die Qualität ihrer Arbeit regelmässig von einer akkreditierten Zertifizierungsstelle überprüfen lassen, einen höheren Taxpunktwert oder eine Qualitätsprämie vereinbaren.

2 BBl Nr. 38 Seiten 7793-7796 (www.admin.ch/ch/d/ff/2008/); BBl Nr. 38 Seiten 7797-7888 (www.admin.ch/ch/d/ff/2008/).

3 BBl Nr. 38 Seiten 7889-7896 (www.admin.ch/ch/d/ff/2008/).

4 http://bit.ly/cTXIzX 